\title{
Esophageal Dysphagia
}

National Cancer Institute

\section{Source}

National Cancer Institute. Esophageal Dysphagia. NCI Thesaurus. Code C113469.

Difficulty in swallowing due to an abnormality in the esophagus. 